\title{
Philosophiques
}

\section{Le concept d'association et l'unité politique : étude critique chez Saint-Simon, Fourier et Marx}

\section{Olivier Perru}

Volume 26, numéro 1, printemps 1999

URI : https://id.erudit.org/iderudit/004965ar

DOI : https://doi.org/10.7202/004965ar

Aller au sommaire du numéro

\section{Éditeur(s)}

Société de philosophie du Québec

\section{ISSN}

0316-2923 (imprimé)

1492-1391 (numérique)

Découvrir la revue

\section{Citer cet article}

Perru, O. (1999). Le concept d'association et l'unité politique : étude critique chez Saint-Simon, Fourier et Marx. Philosophiques, 26(1), 83-108.

https://doi.org/10.7202/004965ar

\section{Résumé de l'article}

Enraciné dans la philosophie politique au XVIIIe siècle français, le concept d'association occupe une place essentielle dans la philosophie de Saint-Simon. Chez cet auteur, l'association a pour objet la production, elle présuppose une organisation intégrée et constitue l'unité de la société. La société organique de Saint-Simon évolue selon un principe de complexification vers un grand but industriel commun. Fourier place aussi l'association comme condition de la coopération, mais elle en est le moteur. L'organisation de l'investissement psychologique dans l'association créerait continuellement la société fouriériste. De Saint-Simon, Marx semble retenir l'idée d'association créant l'organisme productif; il emprunte à Fourier la production d'une société nouvelle collectivisant toutes les dimensions personnelles. Mais l'association devient chez Marx le fruit de la dialectique de l'histoire, dont le moment négatif rend l'organisme collectif à lui-même et lui confère une individualité nouvelle. L'association chez Marx s'impose comme une unité. Chacun participe nécessairement à cette réalité qui doit l'affranchir des limites de sa propre individualité 


\title{
Le concept d'association et l'unité politique : étude critique chez Saint-Simon, Fourier et Marx
}

\author{
OLIVIER PERRU
}

I.S.A.B.

Institut Catholique de Paris ${ }^{1}$

\begin{abstract}
RÉSUMÉ. - Enraciné dans la philosophie politique au XVIII ${ }^{\mathrm{e}}$ siècle français, le concept d'association occupe une place essentielle dans la philosophie de SaintSimon. Chez cet auteur, l'association a pour objet la production, elle présuppose une organisation intégrée et constitue l'unité de la société. La société organique de Saint-Simon évolue selon un principe de complexification vers un grand but industriel commun. Fourier place aussi l'association comme condition de la coopération, mais elle en est le moteur. L'organisation de l'investissement psychologique dans l'association créerait continuellement la société fouriériste. De Saint-Simon, Marx semble retenir l'idée d'association créant l'organisme productif; il emprunte à Fourier la production d'une société nouvelle collectivisant toutes les dimensions personnelles. Mais l'association devient chez Marx le fruit de la dialectique de l'histoire, dont le moment négatif rend l'organisme collectif à lui-même et lui confère une individualité nouvelle. L'association chez Marx s'impose comme une unité. Chacun participe nécessairement à cette réalité qui doit l'affranchir des limites de sa propre individualité.
\end{abstract}

\begin{abstract}
Rooted in the french philosophy of the XVIIIth french century, the concept of association holds an essential place in the philosophy of SaintSimon. In this author, association concerns production, it is generated by an integrated organization and forms unity in the society. In Saint-Simon, the organized society evolves according to a principle of complexification towards a great industrial aim. Fourier equally sets association before cooperation, considering it as a real driving force. According to Fourier, psychological commitment in association should make dynamic any cooperation. Marx possibly deducted from Saint-Simon the idea that association should create the collective organism. He borrowed from Fourier the production of a new society, getting collective the whole human person. But, association in Marx's thought becomes the result of the dialectic of history. A negative phase in this dialectic gives the collective organisme to itself back and confers on it a new individuality. In Marx, association is essential as a unity. Each one necessary takes part in this reality which will make him free from the limits of his own individuality.
\end{abstract}

Le concept d'association tient une place centrale dans l'œuvre de Saint-Simon et de Fourier, et constitue chez ces auteurs le noyau central d'une nouvelle forme de pensée politique, qui garde cependant un enracinement dans la philosophie du XVIII ${ }^{\mathrm{e}}$ siècle $(\mathbb{S} 1)$. En toute rigueur, si l'on souhaite examiner la signification de ce concept dans le courant socialiste français, il faudrait

I. 32, Bd. du port, F-95094 CERGY-PONTOISE.

PHILOSOPHIQUES 26/1 — Printemps 1999, p. 83-108 
aussi examiner l'œuvre de Pierre Leroux. Parce que nous voulions regarder les conséquences ultimes des développements d'une pensée dialectique autour de l'association, il nous a paru plus intéressant de mettre en perspective les deux auteurs qui sont au fondement de ce courant socialiste français, et certaines positions de Marx en la matière. Mais avant de nous lancer dans cet exercice d'analyse et de critique, il faut donner les interrogations philosophiques fondamentales sur la place de ce concept à l'égard de la dimension politique de l'homme : Reflète-t-il une expérience qui permette de fonder cette dimension ? Ou bien, faut-il plutôt considérer l'association comme conséquente à une coopération finalisée et s'inscrivant dans la durée ? En effet, " s'associer » avec d'autres me semble difficilement pouvoir relever d'une expérience première immédiate : l'association suppose une pratique à la fois préalable et durable, elle demande de définir un ou des objets, elle engage vers une finalité. Ainsi, sur un plan critique, la signification du concept d'association implique-t-elle un lien nécessaire avec le concept de coopération ? C'est en ce sens qu'il faut être très attentif aux idéologies socialistes nées au XIX ${ }^{\mathrm{e}}$ siècle, en tant qu'elles semblent poser a priori l'association universelle comme cadre de vie unique et dans la perspective d'une mise en commun des biens nécessaires à la vie. C'est notamment le cas chez Fourier. La lecture et l'analyse comparative de nos trois auteurs pose une autre question : L'association humaine implique-t-elle une structure comparable à celle d'un organisme? En cas de réponse affirmative, on comprendrait alors qu'au lieu d'être un milieu de vie ordonné au Bien de chaque individu, elle devienne une totalité unifiée, conditionnant absolument toute dimension socio-politique de l'individu, et toute fin sociale. Alors, on ne pourrait plus situer l'association dans la ligne d'une véritable expérience humaine, à la fois personnelle et sociale. Il n'y aurait plus de multiples formes d'association, mais une seule, dont l'extension serait celle de la société. Il faudrait rechercher l'intelligibilité du concept d'association au plan de structures sociétales qu'on ne choisit pas, mais qui seraient censées permettre la coopération entre les individus.

Il découle de cette introduction que pour clarifier la signification du concept d'association chez Saint-Simon, Fourier et Marx, nous devons préciser des liens entre trois pôles de l'activité socio-politique de l'homme : l'expérience socio-politique individuelle, les structures sociétales (la ou les forme(s) de l'association) et l'unité politique. Il s'agit de situer l'établissement de l'association par rapport à la société dans son ensemble : est-elle une condition de l'activité dans la cité, ou un fruit de la coopération entre des individus libres et responsables, ou encore le terme d'un processus dialectique ? En essayant de cerner l'intelligibilité du concept d'association chez Saint-Simon, Fourier et Marx, et en tentant de comparer et de critiquer les positions de ces auteurs, il faudra garder présentes à l'esprit les questions fondamentales de toute philosophie politique sur la nature, le rôle et les caractères d'une association dans une société humaine. 


\section{Première approche du concept d'association}

Dans une position réaliste - qui n'est évidemment pas celle des auteurs que nous allons étudier - c'est l'excellence de la personne qui coopère, qui fonde une "philosophie politique ». Au plan sociétal, la découverte du Bien personnel peut à la fois relativiser et enrichir la participation de chacun au Bien Commun de la cité, donc au bien commun de toutes les communautés particulières qui la constituent. Ayant découvert, à travers l'exercice de la volonté, une finalité qui structure mon éthique personnelle, je suis capable de relativiser le Bien de la société, et des diverses communautés humaines auxquelles j'appartiens, dans l'ordre des fins. Mais, je puis aussi y participer plus activement, parce que je saisis dans la dimension communautaire, quelque chose qui me dispose à l'accomplissement de ma vie éthique, et qui me permet d'exprimer la richesse de celle-ci. La koinonia d'Aristote est donc à replacer dans la lumière de l'interrogation de la finalité en matière éthique et politique, puis de la découverte du bien personnel comme finalisant l'agir humain ${ }^{2}$.

Ainsi comprise, l'association serait fondée sur l'expérience de coopération ; elle représenterait en quelque sorte un fruit, une conséquence des efforts de tous les associés dans la conquête du Bien Commun. Impliquant la dimension politique de l'homme, elle serait comme une propriété de la communauté, dans laquelle l'éthique de chacun assume les projets propres communautaires. Elle serait donc à situer du côté de l'unité consécutive à une coopération qui permette un Bien Commun minimal. En effet, ceux qui participent à une communauté quelle qu'elle soit ne peuvent se dire " associés " que s'ils ont l'expérience de la coopération, s'ils participent à l'édification du Bien Commun, et s'ils en reçoivent les fruits. Leur unité ne se mesure pas seulement à leur efficacité, ni à l'expérience de leurs besoins réciproques, mais aux liens de confiance interpersonnels, à leur participation effective à l'ensemble des «projets » de la communauté3.

Ce sont justement ces deux rapports, d'une part de la coopération finalisée qui permet de réaliser l'association, d'autre part de l'association qui tend à l'unité, qu'il importe de bien cerner. L'unité est certes ce qui sera, $a$ posteriori, le caractère le plus tangible pour identifier une association ; mais, si elle apparaît comme une propriété, elle n'est pas pour autant une fin en soi. C'est à chaque type d'association de découvrir et de définir la finalité qui légitime son existence. Il paraît donc difficile de baser toute association sur la perspective de l'unité, ou même sur une perspective plus souple d'harmonie et d'équilibre, a fortiori la société d'un pays dans son ensemble, pour autant

2. S. Vergnières écrit très justement : « Les citoyens sont capables d'agir, non seulement pour subsister, mais pour vivre heureusement. La cité n'est donc une communauté véritablement politique que lorsqu'elle devient une communauté éthique. C'est en accomplissant sa tâche de citoyen que chacun peut faire preuve de ses qualités et goûter le bonheur spécifiquement humain de la vie active ». Le politique et l'éthique entretiennent donc une nécessaire relation réciproque. Vergnières, S., Éthique et Politique chez Aristote, Paris, Essais, PUF, 1995, p. 160.

3. Ainsi, pour $\mathrm{S}$. Vergnières, "la communauté politique est moins une chose organisée qu'un acte commun " (ibid., p. 159). L'unité de la cité est une conséquence de l'apprentissage de cet acte commun par l'éducation à la citoyenneté (Aristote, Politique, II, 5, 1263 b 36-37). 
qu'elle puisse être qualifiée d'association. Si on considère l'unité comme un fruit ultime et conséquent de l'association, il n'est donc pas surprenant que l'association ait été vue par de nombreux auteurs au niveau de l'unité politique. Il semble, de fait, que l'une des tentations les plus fréquentes en philosophie politique soit de confondre la conséquence (de la vie sociétale) que nous venons d'évoquer, c'est à dire l'unité entre les membres associés, et la forme de la communauté, c'est à dire, finalement, sa constitution. Ainsi, pour Platon et pour certains Modernes, l'association comme telle n'est pas le fruit du consentement volontaire actuel des parties en présence. Elle n'appelle pas l'existence de différentes communautés ou corps intermédiaires, mais plutôt une unification de la cité politique. Que cette unification se fasse comme chez Platon dans la lumière d'une causalité exemplaire, ou qu'elle se fasse sous l'impulsion d'une volonté politique unique (idéologique), elle procède souvent de la conviction que c'est le moyen nécessaire pour guider l'ensemble des citoyens vers leur bien. Ce regard, où c'est l'unité politique qui commande l'association et conditionne l'exercice de la vie publique et privée, n'est-il pas, de fait, une projection imaginative de la propriété d'un corps politique structuré sur la finalité de la vie politique?

Par ailleurs, de nombreux auteurs, surtout à partir du XVIII ${ }^{\mathrm{e}}$ siècle, comparent la société à un organisme vivant. Le philosophe politique regarderait ainsi le caractère vital et organique de l'association, sous la dépendance de sa fin propre, ce qui peut paraître séduisant. Mais, il subsiste une question d'objectivité : l'unité qui s'instaure dans un vivant individuel, voire dans une société animale, se retrouve-t-elle analogiquement dans le domaine des sociétés humaines? Quel est le rapport entre les personnes engagées dans cette société et l'unité sociale? En regardant la société humaine dans la perspective d'un organisme, le philosophe politique risque d'assimiler l'association à une dialectique de relations interindividuelles devant tendre vers l'unité.

\section{Association et unité, de Rousseau et de Wolff à Saint-Simon}

L'unité organique de l'association étendue à l'échelle de la société, unité dont va rêver Saint-Simon $(1822)^{4}$, eût des précédents : pour Wolff, l'idée d'unité universelle, pour Rousseau, la construction d'un quasi-organisme social. En 1764, Wolff, dans le Jus Gentium méthodo scientifico pertractatum 5 , recherchera une extension de l'association, telle que les relations internationales, soumises aux principes de la raison, concourent à réaliser une unité résultant d'un quasi-accord. Vieira de Mello a pu écrire : "Wolff reconnaissait donc entièrement, près de trois siècles avant notre époque,

4. Saint-Simon, 1821-22, Système Industriel, Volume V et VI, Les œuvres de SaintSimon et d'Enfantin, Paris, 1865-1878.

5. Wolff, C., 1764, Jus gentium méthodo scientifico pertractatum, J-D. Drake éditions, 2 volumes, Oxford, Clarendon Press, 1934. 
l'existence d'un ensemble de règles qui, au niveau de la cité suprême fictive, ordonnaient les relations entre les états en elle associés ; mais aussi, en raison du caractère fictif de la suprématie de cette association, l'imperfection de cet ordre, ainsi que sa fragilité dérivée de la persistance de l'état de nature ${ }^{6}$.» On peut dire que l'idée d'unité universelle, présente chez Wolff, va connaître des développements inespérés au cours du XIX ${ }^{\mathrm{e}}$ siècle. Son énoncé ultime se trouve dans l'œuvre de F. Laurent (1879) qui, généralisant aux États l'idée de corps social, écrit: "L'unité de l'humanité ne sera pas une unité de coaction, telle que l'unité de l'État ; elle reposera sur le concours volontaire du consentement ; elle sera le résultat du contrat, et non de la loi. En d'autres termes, nous croyons que c'est par la voie de l'association libre que l'unité s'établira, association qui laissera la souveraineté des nations intacte, et garantira plutôt qu'elle n'absorbera leur indépendance ${ }^{7}$. " Cet énoncé semble étendre au plan international ce que Rousseau préconise dans l'État: trouver une forme d'association qui permette l'unité du corps politique, en garantissant la liberté de l'individu. Renvoyons simplement ici à la formulation du "Contrat social " $(I, 6)$ : « Trouver une forme d'association, qui défende et protège de toute la force commune, la personne et les biens de chaque associé, et par laquelle, chacun s'unissant à tous, n'obéisse pourtant qu'à lui-même, et reste aussi libre qu'auparavant $^{8}$. » Chez Wolff comme chez Laurent, cette unité engendrée par l'association revêt une autre dimension d'universalité. Le contrat débouche sur un monde et une culture teintés d'universalisme, dans lesquels coexistent des souverainetés autonomes.

Chez Rousseau, unité ne signifie pas pour autant universalité. La communauté, pour lui, n'a d'une certaine manière rien d'universel : elle revêt un caractère individualisé, et elle traite avec des individus. En précisant $(\mathrm{I}, 5)$ le contenu du contrat : "l'aliénation totale de chaque associé, avec tous ses droits, à toute la communauté ", Rousseau pose les bases de politiques sauvegardant à terme aussi bien l'individualisme que le socialisme. Mais, l'aliénation à la communauté n'est que l'aspect négatif de la mise en commun des personnes et des biens, sous la direction de la volonté générale. Mettre tout en commun implique la constitution d'un nouvel organisme : le corps politique. Deux remarques s'imposent alors :

1) On retrouve l'unité d'un organisme (tout), à partir des individualités distinctes (parties), et ce, au niveau sociétal. Ce n'est évidemment pas sans rappeler les travaux de Buffon. Il faut toutefois noter que "l'idée de nature que se font les philosophes n'est pas, loin s'en faut encore, celle des naturalistes de cette époque9. " Nous devons donc éviter l'amalgame à ce niveau, même si Rousseau, entre autres dans le Discours, fait explicitement référence à Buffon. Par ailleurs, pour Buffon, l'homme

6. Vieira de Mello, S., Civitas maxima : origines, fondements et portée philosophique et pratique du Concept de supranationalité, THÈSe, Université Paris I-SORbONne, I985, P. 484.

7. Laurent, F., Études sur l'histoire de l'humanité, volume I, $2^{\circ}$ Édition, Paris, Marpon et Flammarion, 1879, p. 45.

8. Rousseau, J-J., 1761, Du contrat social, Paris, Garnier Frères, 1954, p. 243.

9. Capitan, C., La nature à l'ordre du jour, 1789-1793, Paris, Kimé, 1993, p. 105. 
comme l'animal est naturellement sociable, c'est le tout social humain qui permet sa vie d'homme. C'est la différence majeure: ce tout social organique, que Buffon considère comme naturel, Rousseau ne fait-il pas que le déplacer dans le domaine de l'artificiel ? De fait, chez Rousseau, cet organisme est d'un nouvel ordre, pure création humaine, non naturelle.

2) Le corps moral et collectif forme une nouvelle individualité qu'il appellera "personne publique ", impliquant comme toute individualité une vie, une volonté. À ce niveau, il est très surprenant de constater que Rousseau considère la société à la fois comme une production de la volonté des hommes et comme un vivant organique qui est une personne publique. Jean-Jacques Rousseau semble avoir une si haute conception de l'homme qu'il confère à l'acte d'association la capacité de donner la vie au corps politique $^{10}$, qui devient alors comme un grand vivant. Le contrat social n'est pas le seul texte de Rousseau qui affirme l'existence du corps politique en tant que vivant et organisé. Dans l'Encyclopédie, à l'article "Économie politique » (1755), Rousseau écrit : «Le corps politique, pris individuellement peut être considéré comme un corps organisé, vivant et semblable à celui de l'homme [...] La vie de l'un et de l'autre est le moi commun au tout, la sensibilité réciproque et la correspondance interne de toutes les parties. Cette communication vient-elle à cesser, l'unité formelle à s'évanouir et les parties contiguës à n'appartenir plus l'une à l'autre que par juxtaposition, l'homme est mort ou l'État est dissous. Le corps politique est donc aussi un être moral qui a une volonté, et cette volonté générale qui tend toujours à la conservation et au bien-être du tout et de chaque partie"."

Pour Saint-Simon, si l'univers est un, cette unité appelle d'une part une unité de la société, d'autre part une unité de méthodologie des sciences. On retrouve dans cette dernière proposition une idée entrevue à la même époque par Lamarck : "Les sciences humaines doivent être construites à l'imitation des autres sciences naturelles, car l'homme n'est qu'une partie de la nature ${ }^{12}$. " Saint-Simon est donc l'initiateur d'une

Io. L'ambiguïté de la pensée de Rousseau — qui montre bien qu'elle n'est pas vraiment " organiciste » - réside toutefois dans le fait que c'est l'homme qui intervient pour créer quelque chose qui est "comme un vivant organique ", mais qui n'est pas un vivant organique réel. De Bonald $(1800,146)$ écrit à ce sujet : "L'homme ne peut pas plus donner une constitution à la société religieuse ou politique qu'il ne peut donner la pesanteur aux corps ou l'étendue à la matière [...]. Bien loin de pouvoir constituer la société, l'homme par son interaction [...] ne peut que retarder le succès des efforts qu'elle fait pour parvenir à sa constitution naturelle. " De Bonald, Essai analytique sur les lois naturelles de l'ordre social, Paris, Bibliothèque nationale, 1800. On retrouve donc la distinction entre ce qui est dans l'intentionnalité de l'homme et ce qui vit réellement. Pour les auteurs du XIX ${ }^{\mathrm{e}}$ siècle, la société est un organisme vivant, naturel et non artificiel.

I I. Rousseau, J-J., "Économie politique ", in L'encyclopédie, ou dictionnaire raisonné des sciences, des arts et des métiers, tome V, Paris, D. Diderot et J. Le Rond d'Alembert, 1755.

I2. Durkheim, E., 1928, Le Socialisme, Paris, Quadrige, PUF, 1992, p. 125. 
science de l'organisme social, véritable " physiologie sociale ", qui a pour objet l'étude des organes du corps social, comme la physiologie animale regarde ceux du corps individuel. Saint-Simon écrit alors : "Le domaine de la physiologie envisagée d'une manière générale se compose de tous les faits qui se passent chez les êtres organisés [...]. La physiologie n'est pas seulement cette science qui, s'adressant à un de nos organes, expérimente sur chacun d'eux [...] pour mieux déterminer les sphères d'activité [...]. Elle ne consiste pas seulement dans cette connaissance comparative qui extrait de l'examen des plantes et des animaux des notions précieuses sur les fonctions des parties que nous possédons en commun avec ces différentes classes d'êtres organisés ${ }^{13}$. Il existe donc une autre science, incluse dans le vocable "physiologie ", qui s'appuie aussi sur des faits pour comparer des organes et déterminer des fonctions : "Elle (la physiologie sociale) plane au-dessus des individus, qui ne sont plus pour elle que les organes du corps social dont elle doit étudier les fonctions organiques, comme la physiologie spéciale étudie celles des individus [...]. La société n'est point une simple agglomération d'êtres vivants dont les actions n'ont d'autres causes que l'arbitraire des volontés individuelles, ni d'autres résultats que les accidents éphémères ou sans importance; la société, au contraire, est surtout une véritable machine organisée dont toutes les parties contribuent d'une manière différente à la marche de l'ensemble. La réunion des hommes constitue un véritable être dont l'existence est plus ou moins vigoureuse ou chancelante, suivant que ses organes s'acquittent plus ou moins régulièrement des fonctions qui leur sont confiées ${ }^{14}$.

Ce texte nous donne une clé pour comprendre la dialectique saintsimonienne. D'abord, Saint-Simon s'appuie sur la démarche expérimentale pour construire une sociologie. Moyennant quoi, il postule, ce que reprendra Durkheim, l'autonomie du social comme réalité vivante et organique, objet d'étude au même titre que le corps biologique. Puis, il est intéressant de voir que, sur cette démarche expérimentale proprement dite, se greffent deux postulats, qui étaient déjà présents chez Wolff et chez Rousseau : d'une part, la recherche de la réalisation de l'unité la plus étendue possible, d'autre part, la formation d'un corps organique réalisant une nouvelle individualité. À part Rousseau qui parle de "corps organisé ", Saint-Simon (avant Spencer), est le premier à décrire réellement la société comme un organisme. Ce nouvel être social implique

I3. Saint-Simon, Physiologie Sociale, Volume X, Les œuvres de Saint-Simon et d'Enfantin, Paris, 1865-1878, pp. 175-176.

I4. Ibid., pp. 176-177. P. Birnbaum $(1975,91)$ parle de «l'organicisme réformiste de Saint-Simon, pour qui la division des fonctions de l'organisme social constitue un facteur de progrès et ne renforce pas simplement la stabilité de la société » (Birnbaum, P., La fin du politique, Paris, Seuil, 1975). De fait, le texte de Saint-Simon montre que l'efficacité de la division des fonctions conditionne la bonne santé du corps social. C'est une vision fonctionnelle de la société, et non pas une vision hiérarchique et formelle comme peut l'être à la même époque l'approche de De Bonald, ou comme celle de Comte tendra à le devenir. 
donc une organisation qui lui soit propre. De même que, chez Lamarck, "l'organisation donne aux êtres vivants la loi interne régissant la possibilité même de leur existence ${ }^{15}$ ", chez Saint-Simon, c'est la loi d'organisation sociale qui rend compte des phénomènes observés dans la société. Or, chez le naturaliste de la Philosophie zoologique, le concept d'organisation appelle la découverte d'une tendance évolutive à travers l'histoire de la vie. Analogiquement chez Saint-Simon, la loi d'organisation sociale appelle un ordre de successions, une tendance au progrès des sociétés. De même que chaque individu vivant peut être replacé dans la perspective transformiste, chaque société peut être expliquée selon le "fait du progrès ", qui relie les diverses formes sociétales. (Ce "fait du progrès » est d'ailleurs hérité de Condorcet.)

L'organisation ne semble pas être synonyme d'association, bien qu'elle nécessite l'association. C'est ce que Saint-Simon expose dans le Système industriel: "L'organisation d'un système bien ordonné exige que ses parties soient fortement liées à l'ensemble, et dans sa dépendance ${ }^{16}$. » On retrouve donc le paradigme dominant l'histoire naturelle au début du XIX ${ }^{\mathrm{e}}$ siècle. Par ailleurs, « il faut que, dans la très grande majorité de la nation, les individus soient engagés dans les associations industrielles plus ou moins nombreuses et liées entre elles [...] ce qui permet d'en former un système général, en les dirigeant vers un grand but industriel commun ${ }^{17}$. » Chez Saint-Simon, les individus doivent être engagés dans des associations, donc vivre une certaine expérience de coopération, certes limitée

I 5. Jacob, F., La Logique du vivant, une histoire de l'hérédité, Paris, Tel, Gallimard, 1970, p. 99. J. Schlanger écrit que cette conception romantique s'oppose à « l'idée d'un agrégat d'atomes ou de rouages ou d'individus isolés, dont la liaison aurait un caractère secondaire en fait et en droit. Et l'on pourrait dire que ce sont les différentes représentations romantiques du mécanisme qui dessinent, a contrario, les principaux traits de l'organisme. Une première contrevalorisation du mécanisme est typifié par la mosaïque[...] : pure juxtaposition d'éléments interchangeables, tous semblables entre eux, indéfinis dans leur nombre et dans leur configuration d'ensemble... À de tels agrégats informes qui ne constituent pas des ensembles réels, l'organisme s'oppose comme l'organisation définie, douée d'une forme propre et par conséquent d'une limite dont les contours expriment l'unité morphologique. L'organisme est une réalité informée. » (Schlanger, J., Les métaphores de l'organisme, Paris, Vrin, 1971, pp. 7 8) Ainsi s'exprime le fait que le début du XIX ${ }^{\mathrm{e}}$ siècle voit la valorisation du vivant comme tout unifié, mais aussi comme système dont la corrélation interne des parties contribue à la bonne marche de l'ensemble. Ce vivant organisé pose des actes successifs pour réagir aux multiples changements d'un milieu en devenir. Il peut prendre de nouvelles habitudes, acquérir de nouveaux organes. C'est alors l'hérédité des caractères acquis (Lamarck, Philosophie zoologique, Paris, librairie Dentu, 1809, I, p. 235). L'organisme peut évoluer ainsi à travers la tendance à la complexification. On voit ce qu'il peut y avoir d'ouverture dans une telle approche : la notion d'effort et de développement individuel dans une durée propre n'est pas restrictive ; elle permet d'intégrer dans une même perspective des dimensions diverses de l'individu, notamment l'esprit. Profitant de cette ouverture, Bergson reprendra à sa manière l'approche de Lamarck. Par contre, l'unité vitale, pour Lamarck, c'est l'individu, sans qu'il se préoccupe en premier lieu de son rapport aux autres individus.

I6. Saint-Simon, Système industriel, Vol. V, p. 16.

I7. Ibid., Vol. VI, p. 185. 
à l'univers professionnel de l'industrie. Mais, l'enjeu est de construire un "système général » en vue d'un " grand but industriel commun ». Le but n'est donc pas de s'appuyer sur toute forme de coopération en vue du Bien Commun ; il est en fait, d'abord, de constituer une association de production qui soit antérieure à la collaboration entre les individus. Elle est perçue comme un moyen privilégié pour construire un corps social unifié s'exprimant à travers l'activité industrielle. Le fait de réduire la société à l'organisme n'entraîne-t-il pas, chez Saint-Simon, une réduction des fins sociales à la production économique ? Comme il l'écrit lui-même, "la politique est la science de la production ${ }^{18}$."

Ainsi, dans l'œuvre de Saint-Simon, l'application de l'idée d'organisation (qui est le concept-clé en histoire naturelle au début du XIX ${ }^{\mathrm{e}}$ siècle) aux sciences sociales semble commander toute l'intelligibilité des concepts d'association, de division du travail social et de solidarité ${ }^{19}$. Au sujet de l'idéologie de Saint-Simon, Durkheim écrit dans Le Socialisme cette phrase synthétique : "Il faut qu'elle (la société) se débarrasse des organes parasitaires que la force de l'habitude maintient encore, et qui absorbent une partie de la vitalité collective, pour ne plus être qu'un système de fonctions économiques, une vaste association de production, et qu'elle s'organise en conséquence ${ }^{20}$. »

Dans le Système industriel, Saint-Simon relativise délibérément la liberté individuelle à l'association politique. Cette liberté ne peut pas constituer une finalité pour les associations humaines, car celles-ci ne sont possibles que grâce à une interdépendance mutuelle qui diminue cette liberté. "On ne s'associe

I 8. Saint-Simon, 1816-17, L'Industrie, Vol. II, Les œuvres de Saint-Simon et d'Enfantin, Paris, 1865-1878, p. 188.

I9. C'est parce que Durkheim rejette cette subordination du social à l'humain, et qu'il veut découvrir la cause de la cohérence globale des relations vitales et sociales découvertes, qu'il veut induire à partir de l'association des individus en société, l'existence d'une individualité vivante nouvelle, et qu'il fait reposer cette induction sur l'analogie biologique (Durkheim, E., 1895, Les règles de la méthode sociologique, Paris, Champs, Flammarion, 1988, p. 195). Cette analogie s'applique éminemment aux questions de la division du travail, et de la "solidarité organique". Dans les sociétés où règne une solidarité organique, et dans lesquelles le principe de division du travail a atteint son évolution optimale, chaque organe social accomplit une fonction spécialisée et déterminée, dans l'interdépendance et la complémentarité. Durkheim écrit, dans La division du travail: "Les éléments sociaux ne sont ni juxtaposés linéairement comme les anneaux d'un annelé, ni emboîtés les uns dans les autres, mais coordonnés et subordonnés les uns aux autres autour d'un même organe central qui exerce sur le reste de l'organisme une action modératrice. Cet organe lui-même n'a plus le même caractère que dans le cas précédent (la solidarité mécanique), car, si les autres dépendent de lui, il en dépend à son tour. Sans doute, il a bien encore une situation particulière et, si l'on veut, privilégiée ; mais elle est due à la nature du rôle qu'il remplit, et non à quelque cause étrangère à ses fonctions, à quelque force qui lui est communiquée du dehors. " Pour Durkheim, les évolutions sociales montrent que toute société est appelée à passer d'une organisation politico-sociale segmentaire, à une organisation beaucoup plus « évoluée ", basée sur le milieu professionnel, impliquant la spécialisation et la division du travail, dans la coopération (Durkheim, E., 1893, La division du travail social, Paris, Quadrige, PUF., 1991, p. 161 et 93-94). Mais, cette évolution sociale implique une tendance à l'organisation dont on ne découvre pas la cause efficiente. De l'analyse des faits sociaux, on retomberait (comme chez Saint-Simon, Comte ou Spencer) sur la tendance (lamarckienne) de la vie à la complexification. Or, dans le domaine socio-politique, peut-on envisager une telle tendance, qui semble spontanée, à l'ordre et à la cohésion sociale ?

20. Durkheim, E., Le Socialisme, p. 168. 
point pour être libre [...]. La vraie liberté ne consiste pas à rester les bras croisés dans l'association ; un tel penchant doit être réprimé partout où il existe; elle consiste, au contraire à développer sans entraves, et avec toute l'extension possible, une capacité temporelle ou spirituelle utile à l'association ${ }^{21}$. »

Finalement, l'association signifie-t-elle, pour Saint-Simon, une association de production déterminée, ou la société dans son ensemble, constituée en association de production ? Comme chez Fourier, il semble que l'association de production soit la fin des potentialités des individus et qu'elle se concrétise dans une structure relativement proche de l'individu (entreprises, communes, etc.), tout en ayant vocation à s'étendre à l'ensemble de la société. En effet, dans le Système industriel que projette Saint-Simon, l'engagement des individus se fait dans des associations industrielles qui sont liées entre elles au plan professionnel, dans un système général de même nature. Cette combinaison d'associations peut, au départ, être relativement lâche et correspondre à des échanges économiques plutôt qu'à une structure collectivisée. Ainsi Saint-Simon, par l'association industrielle, et Fourier, par le phalanstère, maintiennent encore des structures intermédiaires entre l'individu et le système politique, même si le but ultime est la réalisation de l'association politique, pour l'un, et de l'unité universelle, pour l'autre. Ce projet de commencer par instaurer des associations proches de l'individu (en vue d'une intégration dans une unique organisation) n'existera plus réellement chez Pierre Leroux : peu à peu, les socialistes français vont mettre un ordre socioéconomique entre l'individu-partie et l'organisme-tout. Plus tard, Marx (1844) fera de l'être social le constitutif propre de l'individu, supprimant ainsi la distance entre l'individu et la société, faisant de la partie la totalité dans une unité idéale. C'est probablement la différence la plus forte avec les socialistes français, pour qui l'unité collective organisée ne suffit pas à résumer la participation de chaque individu.

Saint-Simon tient beaucoup à ce niveau de la participation propre de chacun à l'activité du corps social. G. Ionescu écrit : "Saint-Simon pensait que, par l'ultime acte de leur engagement dans la société de production, tous les producteurs pourraient, à travers leur habileté, leur savoir-faire et leur connaissance, se joindre directement aux processus de décision et de stratégie politique, car ils se trouveraient désormais inévitablement au centre même des choses. [...] Les producteurs s'organisent eux-mêmes en corporations, entreprises, syndicats ou communes qui peuvent, collectivement, offrir ou empêcher leur participation à la production ${ }^{22}$. " Ainsi, l'association s'étend d'organisations créées par un groupe d'individus à toute la société d'un État et l'on ne peut être véritablement associé que si l'on met en œuvre ses capacités spécifiques : les inégalités et

2I. Saint-Simon, Système industriel, Vol. V, p. 15.

22. Ionescu, G., La pensée politique de Saint-Simon, Paris, Bibliothèque sociale, AubierMontaigne, 1979, p. 45. 
les différences naturelles conditionnent le bon fonctionnement des divers organes de la société. Une telle vision est exprimée dans le manifeste saintsimonien, cité par L. Reybaud : "Le système de communauté des biens s'entend universellement du partage égal entre tous les membres de la société, soit du fonds lui-même de la production, soit du fruit du travail de tous. Les Saint-Simoniens repoussent ce partage égal de la propriété [...] car, ils croient à l'inégalité naturelle de l'homme, et regardent cette inégalité comme la base même de l'association, comme la condition indispensable de l'ordre social. Ils repoussent le système de la communauté des biens [...]. Ils demandent que tous les instruments de travail, les terres et les capitaux qui forment aujourd'hui le fonds morcelé des propriétés particulières, soient exploités par association et hiérarchiquement, de manière à ce que la tâche de chacun soit l'expression de sa capacité, et sa richesse, la mesure de ses œuvres ${ }^{23}$. »

Nous pouvons ainsi résumer les positions de Saint-Simon en matière d'individualité et d'association :

1) Il semble que le sens de ce concept soit celui d'association de production. Les individus, avec leurs richesses et leurs potentialités, restent identifiables en amont et en aval de l'association (ce qui constituerait une similitude avec Rousseau). Leur participation effective ne fait aucun doute, mais l'expérience requise dans de nombreux écrits de Saint-Simon semble relever d'un développement de facultés individuelles utiles à l'association (Saint-Simon analyse par exemple, dans L'organisateur, les conséquences pour la société de la disparition de telle ou telle catégorie de personnes, en fonction de leur utilité). Ainsi, l'expérience en question serait à situer au plan d'une dialectique entre l'individu et l'association, mais il est peu question de la nature des relations humaines développées dans l'association ; on peut simplement penser qu'elles sont d'abord d'ordre économique. De plus, la finalité envisagée, le "grand but industriel commun ", même s'il recouvre l'ensemble des activités humaines et pas seulement l'industrie au sens restreint, est sans doute insuffisant pour le maintien d'une société culturellement homogène et solidaire, de même que nul ne peut affirmer qu'un tel but dispose tout citoyen à se réaliser pleinement, c'est-à-dire à être heureux. Non seulement, l'expérience envisagée nous semble purement hypothétique, mais elle serait insuffisante pour fonder véritablement une dimension politique de la personne humaine.

2) Du point de vue de la forme de la société envisagée, l'association de production impliquant l'organisation constitue la cellule de base de la société qu'imagine Saint-Simon. Elle est vue comme une condition du bon exercice de la coopération, au lieu d'en être la conséquence. On ne commence pas par collaborer, mais on commence par s'organiser en association. Cela pourrait paraître logique, dans la mesure où une structure de droit est nécessaire, mais souvenons-nous que Saint-Simon refuse "l'arbitraire des volontés individuelles ", et subordonne la fonction sociale à l'intégration dans un cadre

23. Reybaud, L., Études sur les réformateurs ou socialistes modernes, Saint-Simon, Charles Fourier, Robert Owen, Paris, Guillaumin, 1849, p. 119-121. 
général, dans le «système ». Ce renversement entre l'expérience sociopolitique individuelle et les structures sociales et l'unité politique, semble caractéristique des mouvements socialistes : non seulement la société en tant qu'elle existe est antérieure à l'individu, mais dans sa forme, elle implique une organisation qui a son autonomie à l'égard des individus. Donc, chez SaintSimon, c'est l'association préexistante des individus qui permettra la production en commun, et non l'inverse.

3) Comme chez Condorcet (et, d'une autre manière, comme chez Lamarck), la société organique de Saint-Simon progresse et évolue selon une tendance à la complexification. Cette tendance fait de la société un grand vivant qui est de plus en plus unifié, et implique ce que Durkheim appellera la solidarité organique. Cette unité, toutefois, chez Saint-Simon, laisse une grande place à la vie de l'individu, à l'expression de ses capacités et à sa rétribution. Ainsi, l'unité de la société saint-simonienne est peut-être plus une condition sine qua non pour entreprendre une œuvre commune que le caractère d'une société accomplie. Elle est donc une unité de fonctionnement qui permet l'efficacité et se mesure dans l'ordre de l'utile, mais ne résume pas toute la vitalité et la richesse collective. Le progrès social accroît cette unité. Toutefois, il semble que s'il existe une fin de la société, "un grand but industriel commun ", elle n'exclut pas à terme l'autonomie individuelle, bien que dans l'exercice social les potentialités individuelles doivent s'ordonner à une unité commune.

\section{Fourier, le prophète de l'unité universelle}

Dans la Théorie de l'unité universelle (1822), Charles Fourier fait figure de prophète d'un universalisme associatif, axé à la fois sur la psychologie et sur l'économie. L'association y est conçue comme un système et un ensemble de mécanismes, comme une machine dont la réalisation paraît conditionner totalement le «sort du genre humain ${ }^{24}$ ». Cette association utilise les tendances affectives en vue d'un Bien de la société, qui est lui aussi placé à la fois au niveau de l'économie d'une part, et de l'affectivité passionnelle d'autre part. Fourier avertit ses lecteurs que "l'instinct les guidera dans cette étude séduisante pour quiconque envisage le but et les fins, qui sont d'arriver au triplement de fortune et à la perfection de santé, pour l'exercice continuel des plaisirs et leur variété infinie ${ }^{25}$. »

Au plan psychologique, il s'agit de " développer les passions par séries de groupes contrastés ${ }^{26}$ ", " d'utiliser les passions de chaque individu, faire emploi de tous les caractères bons ou mauvais, rapprocher les antipathiques

24. Fourier, C., Théorie de l'unité universelle, tome VI, Paris, Société pour la propagation et la réalisation de la théorie de Fourier, 1822, Initial, art. I.

25. Ibid., Initial, art. I.

26. Ibid., I, art. I.

27. Ibid., I, art. III, 10. Le développement des passions, préconisé par Fourier, eût des 
par utilité réciproque. ${ }^{27} »$ Fourier ne croit pas à l'existence d'une capacité individuelle d'ordre moral à assumer des responsabilités en société, dans une perspective de philanthropie ou d'intérêt général, indépendamment des passions. Pour rétablir l'harmonie sociale, il faut d'abord résoudre ce problème des passions. On les utilisera pour absorber les rivalités individuelles, et permettre l'attraction passionnée comme base des rapports professionnels et sociaux. L'idée d'utiliser les passions au lieu de chercher à les détruire est apparemment judicieuse, à condition qu'on les subordonne aux facultés spécifiques de l'homme. Mais, chez Fourier, le désir est d'abord psychologique et passionnel, la volonté humaine paraît entièrement mue par les passions et dépendante à leur égard. La seule solution est alors de développer ces dernières et de chercher à leur niveau un équilibre entre les individus : le monde de Fourier est un monde de passions! Fourier écrit :

«Une planète peut être harmonique en sa marche matérielle, et subversive en sa marche sociale : tel est l'état de notre globe, ainsi que de toutes les jeunes planètes ; elles sont sujettes à un régime social subversif, jusqu'à ce qu'elles aient découvert les lois de l'harmonie sociétaire des passions; découverte où elles ne peuvent parvenir qu'en créant d'abord les éléments d'association, la grande industrie, les sciences et les arts ; puis en suspectant les systèmes de philosophie et de contrainte, et en étudiant par analyse et synthèse de l'attraction, les vues sociales de la nature, le mécanisme des séries contrastées, qui est le seul conforme aux dispositions de l'univers, et le seul naturel, s'il y a unité de système dans la nature ${ }^{28}$. »

On retrouve donc ici un lien entre l'unité de la nature et l'unité de la société29, lien que Saint-Simon avait déjà pressenti. Si la nature est une, la société doit tendre à la même unité, en s'inspirant du fonctionnement des vivants. Chez Saint-Simon, c'était l'analogie de l'organisme qui dominait ; ici, ce sont les lois physiques que Fourier tente d'appliquer par analogie, dans un essai de «psychologie sociale ${ }^{30}$."

lettre à Mirabeau, Paris, Garnier-Flammarion, 1981). Précédents au XVIIIe siècle chez Vauvenargues, Diderot, Helvétius. Citons simplement deux phrases de Vauvenargues, dans Introduction à la connaissance de l'esprit humain, p. 200 et 404 : "La force de l'âme est dans le cœur, c'est à dire dans les passions ", et " une vie sans passions ressemble bien à la mort » (Vauvenargues, Introduction à la connaissance de l'esprit humain,

28. Ibid, I, art. IV.

29. Dans la Théorie de l'unité universelle, outre le principe ultime de l'attraction et de la justice distributive, "l'Universalité de providence et l'Unité de système " sont les attributs du Dieu de Fourier, posé comme nécessité logique et garant du système. Il est donc principe d'unité de la nature. L'unité sociétale s'inscrit dans une perspective générale d'unité de l'homme et de l'univers, de l'esprit et de la vie, du biologique et du physique. Pour synthétiser, on peut dire que Fourier voit l'unité de système entre le naturel et le spirituel comme conséquence de la loi générale d'attraction. Dans la Théorie des quatre mouvements, il écrit : " Je reconnus bientôt que les lois de l'attraction passionnée étaient en tout point conformes à celles de l'attraction matérielle[...], qu'il y avait unité du système de mouvement pour le monde matériel et spirituel. Je soupçonnais que cette analogie pouvait s'étendre des lois générales aux lois particulières ; que les attractions et propriétés des animaux, végétaux et minéraux étaient peut-être coordonnées au même plan que celles de l'homme et des astres » (Fourier, C., 1808, Théorie des quatre mouvements, Euvres complètes, tome I, Paris, 1842, p. 12).

30. Concernant l'universalisation des lois de la physique de Newton, C. Morilhat écrit : « Fourier achève ce que Newton avait esquissé en faisant de l'attraction le principe d'intelligibilité 
Se voulant lieu d'expression de la nature et de la vie, le monde phalanstérien sort délibérément d'une perspective morale et d'une perspective politique classiques. Le rôle dynamique des passions dans cette société nous semble alors à juste titre infra-humain, bien proche de la vie animale ; cela correspondrait à la recherche de nouveaux modes de manifestation de l'essence même de la vie dans les rapports sociaux. Un commentaire de P. Bruckner (1975), dans son livre sur Fourier, fait comprendre ce que voudrait être cette tentative sociale : "Le phalanstère ne se présente pas comme un projet politique classique (encore guidé par des critères d'efficacité, de rendement, et donc de pouvoir), mais comme un espace où vont se passer certaines choses, où vont se côtoyer certaines intensités [...]. Pour l'aventure phalanstérienne, il n'y a pas d'échec ou de triomphe, mais seulement un nombre illimité d'excès, de tentatives toujours réitérées, la manifestation gratuite et exemplaire de la vie ${ }^{31}$. » Le problème est alors de savoir si la passion au sens que lui donne Fourier est le meilleur moyen d'exprimer une énergie vitale proprement humaine dans la société.

Néammoins, selon Fourier, l'utilisation des passions et des structures tempéramentales devrait permettre l'intégration des individus dans la "phalange de séries industrielles" en lien avec le développement de l'industrie, des sciences et des arts Cela permettrait de constituer un système conforme à la nature. L'attraction à l'égard "du travail agricole et manufacturier ${ }^{32}$ » et l'harmonie sociétaire, basées sur des caractéristiques individuelles, intégrées dans des structures de progrès scientifique et technique, aboutiraient à la synthèse associative qu'est la société fouriériste. Comme chez Saint-Simon, on y trouve les caractéristiques essentielles du socialisme français à sa naissance : association volontaire d'individus dans un système organique, impliquant mise en commun et solidarité, lié au développement de l'industrie (même si Fourier met le travail agricole en valeur davantage que Saint-Simon ) et du savoir scientifique.

Toutefois, chez Fourier comme chez Saint-Simon, l'association reste un moyen au service de l'individu. Mais, et c'est la différence avec Saint-Simon, la collectivisation ne se limite pas à l'outil de production, mais elle atteint la vie toute

universelle, en unifiant l'ensemble du savoir. À l'auteur des Principes mathématiques de la philosophie naturelle, l'inventeur emprunte essentiellement le mot attraction ". Quant au rapport entre les êtres naturels, les vivants et la société des hommes, Fourier les saisit dans une même continuité. Il aurait été influencé ici par le "continuisme " des naturalistes du XVIII siècle. De plus, résumer comme nous l'avons fait la pensée saint-simonienne par le concept d'organisme social ne signifie pas que la notion d'attraction newtonnienne en soit absente. Bien au contraire, Saint-Simon, comme Fourier, utilise cet universel au fondement de sa vision sociétale. Mais, alors que chez Saint-Simon c'est l'autonomie de l'organisme qui résume le mieux la société (comme d'ailleurs le monde vivant), chez Fourier, c'est la loi de l'attraction qui assure « l'intelligibilité de tout le réel », du « matériel » au « passionnel » (Morilhat, C., Charles Fourier, imaginaire et critique sociale, Philosophie, Méridiens Klincksieck, 1991, p. 99 et 33).

3I. Bruckner, P., Fourier, Paris, Seuil, 1975, p. 159.

32. Fourier, C., Théorie de l'unité universelle, I, art III, 10. 
entière, et touche les individus dans leur liberté personnelle : liberté dans les relations humaines, liberté dans l'organisation de son temps et de sa vie. L'association au sens de Fourier requiert certes, dans sa mise en place, une volonté libre. Mais, choisit-on vraiment la série ou le groupe dans lequel on va vivre, ou y est-on affecté par une sorte de déterminisme psychologique ? Fourier écrit : "La distribution par séries contrastées, pouvant seule utiliser les passions de chaque individu, faire emploi de tous les caractères bons ou mauvais, rapprocher les antipathiques par utilité réciproque, ils reconnaitraient que cet ordre est gage de bonheur individuel, en ce qu'il assure à chacun l'essor des passions 33 ». Si l'homme vit au niveau d'une mécanique passionnelle, comme Fourier le suppose, le système peut "fonctionner ", mais suppose l'absence de toute recherche personnelle qui soit au delà du niveau psychologique. En ordonnant l'organisation sociale au bonheur de chacun, Fourier veut faire accepter sa détermination par séries contrastées (reposant sur une synthèse de l'attraction psychologique et de la nécessité économique). Retenons que, comme chez SaintSimon, c'est, au terme du processus, l'intérêt et le bonheur de l'individu qui sont envisagés, même si la forme de société envisagée et son but paraissent tout à fait impropres pour permettre effectivement le bonheur de chacun.

Au delà des conditions de mise en place, sur lesquelles il s'étend longuement, comment Fourier voit-il cette association? Il la considère d'abord comme une sortie de l'état civilisé, " puisque l'état civilisé n'est autre chose que l'industrie morcelée et opposée au sens commun en fait d'économie ». Il ne s'agit donc pas moins que d'installer les phalanstères, à partir de communautés existantes. " Un canton ou village de 300 familles n'aurait qu'un grenier ou qu'une cave bien soignée, au lieu de 300 greniers et caves mal tenus ; il n'aurait qu'une cuisine préparant en divers degrés, au lieu de 300 feux occupant 300 ménagères [...]. Enfin, il aurait l'unité d'action dans la haute ou la basse industrie, dans le soin des forêts, les travaux d'irrigation, le régime des chasses $[\ldots]^{34}$. » D'une certaine manière, les structures actuelles, en matière de distribution, de loisirs, autant qu'en matière d'administration du territoire et d'économie régionale, ont partiellement réalisé le rêve de Fourier. Mais celuici allait beaucoup plus loin. Il préconisait « d'étendre le lien sociétaire aux plus grandes masses locales, [...] d'assembler des familles inégales en fortune et en tous sens, pour assurer la variété des travaux et la coopération de chacun à divers détails ; $[. .$.$] d'associer les dites masses dans leur trois facultés$ industrielles, capital, travail et talent ; de trouver un moyen de répartition proportionnelle aux 5 facultés, de manière à satisfaire chaque individu, homme femme ou enfant, dans l'allocation des trois sortes de dividendes; d'opérer l'association en passionnel comme en matériel ; de concilier les classes antipathiques en les rendant nécessaires les unes aux autres ${ }^{35}$. " Ce texte mérite un examen attentif, car il propose un renouvellement, qui tient à la fois du contrat social (chacun s'unit à tous et n'obéit qu'à lui-même...) et de la

33. Ibid., I, art. III, 10.

34. Ibid., I, art. II.

35. Ibid., I, art II, note 3. 
production d'une nouvelle société. À terme, la famille disparaît, il reste des individus rémunérés proportionnellement à leurs apports ; l'association est tributaire, pour sa survie, du développement de ces «trois fonctions primordiales » de Fourier, l'exploitation, la consommation, la distribution. Le thème de la conciliation des antipathies permet aussi la pérennité de la société. Le premier obstacle à cette conciliation, le vol et toute forme de malhonnêteté (incluant aussi, finalement, la propriété privée des moyens de production), est impossible dans le socialisme fouriériste ; en effet, ce qui serait dérobé à la collectivité ne peut être revendu ou réutilisé officiellement, celui qui s'enrichit frauduleusement ne peut qu'être découvert et puni. D'une certaine manière, les régimes communistes ont utilisé ce principe dans leur économie, surveillant de près la collectivisation de la production et de la distribution. Mais, à la différence de l'idéologie communiste ultérieure, l'utopie fouriériste maintient "la cupidité individuelle, les inégalités de fortune, de rang et de lumière ${ }^{36}$ " comme moyens d'organisation de l'ordre sociétaire dans la série contrastée. En effet, comme chez Saint-Simon, le système vise à une rétribution satisfaisante de chaque individu, dont, en outre, il recherche le " bonheur " psychologique.

Il semble que l'on retrouve chez Fourier l'inversion entre expérience de coopération et association plus nettement que chez Saint-Simon. C'est la mise en association qui va dynamiser la coopération au plan productif, et non l'association qui va se construire peu à peu à partir de la coopération. La structure sociale va ici plus loin que la constitution d'un organisme productif : elle touche aux personnes dans leur intégrité, leurs structures tempéramentales. C'est une vie commune qu'elle envisage, et pas seulement une mise en commun des moyens de production. De ce fait, l'unité qu'elle vise devient corrélative à l'harmonie entre les individus et à leur bonheur. Ainsi, elle est plus qu'une simple condition, elle est étroitement liée à la finalité d'épanouissement individuel et social à laquelle elle tend. Par ailleurs, par rapport à Saint-Simon, cette unité n'est plus de nature organique, au sens biologique, mais de nature psychologique.

Nous pouvons maintenant conclure sur l'intelligibilité du concept d'association chez Fourier. Comme nous l'avons vu, la question de l'expérience fondatrice du social est centrale chez cet auteur. Elle veut tenir compte de tout le donné humain dans la construction de la société, mais est projetée dans l'hypothétique. Peut-on encore parler d'expérience lorsque l'on nie complètement le donné social existant ? Comment savoir si l'investissement de la psychologie humaine dans l'association, telle que la préconise l'auteur (même en supprimant toutes les fantaisies arithmétiques de l'organisation du phalanstère), permet de construire l'association ? Parmi les expériences inspirées de Fourier au XIX ${ }^{\mathrm{e}}$ siècle, certaines ont réussi à durer quelques années et à transformer la condition ouvrière d'un secteur professionnel donné (dans la région de Saint Quentin notamment); mais aucune n'a débouché sur une 
transformation sociale à long terme. Nous avons mentionné que l'association est une structure concrète intégrant toutes les dimensions de la vie de l'individu. Correspondant à la mise en œuvre de lois naturelles, elle se constitue en groupes à l'intérieur desquels les relations de travail entraînent aussi bien la coopération que la concurrence. L'association de Fourier est aussi un enchaînement de structures, fondé sur une anthropologie et même sur une philosophie de la nature, et ayant vocation à déboucher sur une unité sociétale universelle. Comme l'écrit C. Morilhat, "l'association se substitue au morcellement civilisé37». Enfin, l'organisation de l'association, basée sur le développement en séries, est un emprunt à la biologie plus qu'aux mathématiques. Si l'on peut parler d'organicisme social chez Fourier, c'est en lien avec cette organisation «naturaliste » de la société ; mais c'est aussi un organicisme psychologique. Par ailleurs, le bonheur de la personne à terme parait extrêmement lié à l'unité et à l'harmonie dans la société : en cela, Fourier annonce l'assimilation marxienne de l'individu à la société. La remise en cause de l'autonomie morale de la personne est l'aspect le plus dramatique de l'association fouriériste : peut-on encore parler de bonheur, à ce prix ?

\section{Marx, ou la communauté constituant l'individu}

Un apport capital des précurseurs du socialisme français est l'introduction du thème de la "production " d'une nouvelle forme de société, par des structures de coopération et de participation, et par une expansion industrielle. Toutefois, si l'on prend l'exemple de Fourier, la lutte des classes n'existe pas puisque les antagonismes préexistants sont utilisés pour dynamiser et unifier l'ensemble. Ainsi, l'aspect utopique de Fourier est révélé par l'idéalisation des rapports humains dans l'association, tout en ramenant l'homme à la psychologie des passions. Mais, pour Fourier comme pour Marx, l'association a pour objet immédiat la production à travers une organisation impliquant un certain degré de collectivisation. Marx a bien saisi (Manuscrits de 1844) que le socialisme de Fourier cherche à abolir un mode particulier du travail (essence de la propriété) : "Le travail nivelé, morcelé, et par suite non-libre, est saisi comme la source de la nocivité de la propriété privée, et de son existence aliénée à l'homme ${ }^{38}$. " Au delà de Proudhon (qui refuse le capital, mais accepte la mise en œuvre de l'initiative individuelle) et de Fourier (qui refuse le morcellement du travail, des moyens de production et de distribution, mais accepte le capital privé), Marx veut réaliser le communisme comme "expression positive de la propriété privée abolie ». Pour saisir la signification de l'association dans Les manuscrits de 1844 et dans Le Capital, il faut comprendre ce " rapport de la communauté au monde des choses " qu'est la propriété privée ; cela nécessite au préalable de préciser le regard de Marx sur le rapport entre l'individu et la communauté, rapport qui se précise dans les conceptions marxiennes de la

37. Morilhat, C., Charles Fourier, Imaginaire et critique sociale, p. 141.

38. Marx, K., 1844, Les manuscrits de 1844, Paris, Éditions sociales, 1962, p. 84-85. 
coopération et de la division du travail. Quelle expérience humaine et quels processus sociaux la coopération recouvre-t-elle selon Marx ? Quel est le lien entre la coopération et l'association selon cet auteur ?

Dans les chapitres XIII et XIV de la quatrième section du Capital (Livre I, 1867), Marx cherche à démontrer que la coopération tend à réaliser un organisme social, qui n'est pas pleinement lui-même, qui ne constitue pas un véritable corps avec des membres associés, tant qu'il est inféodé au mode de production capitaliste. La coopération comme telle est essentiellement, pour Marx, un mode de fonctionnement et de production ; elle s'évalue en termes de productivité ; elle n'appelle pas, chez Marx, des relations et des finalités de nature éthique. "Quand plusieurs travailleurs fonctionnent ensemble en vue d'un but commun, dans le même processus de production, ou dans des processus connexes, leur travail prend la forme coopérative [...]. Il s'agit non seulement d'augmenter les forces productives individuelles, mais de créer par le moyen de la coopération, une force nouvelle ne fonctionnant que comme force collective ${ }^{39}$. " La coopération crée une nouvelle capacité de travail, non seulement par la fusion des diverses forces individuelles, mais aussi par l'émulation due au « contact social » entre les divers partenaires. Pour Marx, cette conjonction des efficacités individuelles est réellement productive d'un nouvel être social qui relève en dernier lieu de l'espèce humaine. C'est là son premier effet, avant même l'œuvre exécutée au profit du capital. "En agissant conjointement avec d'autres, dans un but commun ", écrit encore Marx, "et d'après un plan concerté, le travailleur efface les bornes de son individualité, et développe sa puissance comme espèce ${ }^{40}$. »

Cependant, cette coopération entre ouvriers salariés est tributaire du " commandement du capital », " condition réelle de la production ${ }^{41}$ ». Du fait même que la coopération des travailleurs crée une entité sociale encore en puissance, cette dernière est appelée à devenir le cadre normal de toute coopération. Tant que ce n'est pas le cas, Marx constate qu'elle se traduit par une force totalement dépendante et conséquente de la direction capitaliste, quoiqu'organique : Marx introduit ici l'aliénation. Il écrit :

La coopération des ouvriers salariés n'est qu'un simple effet du capital qui les occupe simultanément. Le lien entre leurs fonctions individuelles et leur unité comme corps productif se trouve en dehors d'eux, dans le capital, qui les réunit et les retient. [...] En tant qu'ils coopèrent, ils forment les membres d'un organisme actif, ils ne sont même qu'un mode particulier d'existence du capital ${ }^{42}$.

La dialectique hégélienne du maître et de l'esclave introduit dans la thèse de l'organisme collectif, vu comme nouvelle individualité de

39. Marx, K., 1867, Le Capital, Quatrième section, Livre I, Paris, Champs, Flammarion, 1985, Ch. XIII, p. 242.

40. Ibid., Ch. XIII, p. 244.

4I. Ibid., Ch. XIII, p. 245.

42. Ibid., Ch. XIII, p. 246-247. 
production, l'antithèse de la totale dépendance à l'égard du capital, dans un devenir, en amont et en aval du processus de production :

La force productive que des salariés déploient en fonctionnant comme travailleur collectif, est par conséquent, force productive du capital. Les forces sociales du travail se développent sans être payées, dès que les ouvriers sont placés dans certains cadres, et que le capital les y place. Parce que la force sociale du travail ne coûte rien au capital, et que, d'un autre coté, le salarié ne la développe que lorsque son travail appartient au capital, elle semble être une force dont le capital est doué par nature, une force productive qui lui est immanente ${ }^{43}$.

La conscience universelle de cette potentialité et de cette aliénation prépare l'avènement de la communauté réelle. Cette interprétation d'une situation sociale montre que Marx pense avoir trouvé la solution : il faut que l'entité nouvelle qui se dégage de la conscience de cette force productive se structure en communauté, afin de libérer les individus de leurs limites et des aliénations qu'ils rencontrent.

Marx et Engels maintiennent (comme Saint-Simon, et beaucoup plus que Fourier) l'analogie entre l'organisme social et l'organisme vivant, comme composition d'individualités à l'origine distincte. Pour expliciter l'effet de la coopération simple, Marx évoque « les bancs énormes de corail, surgissant du fond de l'océan, formant des îles et de la terre ferme, bien que chaque individu qui contribue à les construire soit faible, imperceptible et méprisable ${ }^{44}$. » La coopération produit dans ce cas une association en même temps qu'une œuvre destinée à devenir cadre de vie. Le regard marxien sur la division du travail s'inspire encore de cette analogie, et le texte de Marx décrivant le " travailleur collectif » dans la manufacture, au chapitre XIV de la quatrième section du Capital, n'est pas sans rappeler les remarques de Milne-Edwards ${ }^{45}$ sur la

43. Ibid., Ch. XIII, p. 247.

44. Ibid., Ch. XIII, p. 267.

45. Traitant de l'organisme, Milne-Edwards établit clairement un processus vital entre les actes posés par le vivant et la forme des organes d'une part, et l'établissement d'une plus ou moins grande complexité organique d'autre part. On peut l'analyser ainsi : chaque organe est doué d'une perfection plus ou moins grande, et d'une spécialisation qui le rend distinct des autres par la fonction et l'anatomie. Au niveau de l'action de chaque organe, l'auteur situe alors sa perfection et la qualité du produit qui en est le fruit. Le vivant semble agir sous l'impulsion d'un principe moteur impliquant intrinsèquement la division du travail. Finalement, c'est l'observation des divers organismes, du moins parfait vers le plus parfait, qui permet une sorte d'induction de ce principe, qui semble se situer à la fois dans la durée et dans l'immanence des vivants successifs.En effet, on part des divers degrés de perfectionnement des organismes, du nombre, de la dissemblance et de la spécialisation plus ou moins grande de leurs parties, etc. C'est à partir de ces propriétés que l'auteur tente d'induire le principe de division du travail qui paraît connexe à la tendance vitale à la complexification. Toutefois, il faudrait s'interroger sur la validité de cette induction qui suppose a priori le lien entre les organismes, dans la durée.

En fait, Milne-Edwards développe l'intuition de Lamarck en la déclinant dans le rapport des organes entre eux, ce qui implique de considérer la tendance à l'organisation comme tendance à la spécialisation des fonctions, mais aussi l'individu-produit et résultat comme un ensemble d'organes spécialisés en interdépendance mutuelle. C’est vraiment là la conséquence ultime de la pensée de Milne-Edwards. L'efficience de la machine vivante crée elle-même des fonctions, et donc des organes, de plus en plus individualisés, mais aussi un tissu de relations et de contrôles réciproques entre ces fonctions et ces organes. 
division du travail organique $(1827,1843,1857)^{46}$. C'est la division du travail qui crée l'organisme qu'est la manufacture ; son unité se prend d'individualités initialement distinctes. Chaque individu élémentaire évolue en organe relatif à une fonction.

C'est le travailleur collectif formé par la combinaison d'un grand nombre d'ouvriers parcellaires, qui constitue le mécanisme spécifique de la période manufacturière. [...] Le travailleur collectif possède maintenant toutes les facultés productives au même degré de virtuosité, et les dépense le plus économiquement possible en n'employant ses organes, individualisés dans des travailleurs ou des groupes de travailleurs spéciaux, qu'à des fonctions appropriées à leur qualité. En tant que membre du travailleur collectif, le travailleur parcellaire devient même d'autant plus parfait qu'il est plus borné et plus incomplet. L'habitude d'une fonction unique le transforme en organe infaillible et spontané de cette fonction ${ }^{47}$.

L'idée de travailleur parcellaire est présente chez Marx, mais s'appuie sur un texte des Fondements de la philosophie du droit de Hegel (1821) : "Puisque le but des individus est médiatisé par l'universel, qui leur apparaît ainsi comme moyen, il ne peut être atteint par eux que dans la mesure où ils déterminent euxmêmes leur volonté et leur action de manière universelle, et se transforment en maillon de la chaîne de cet ensemble ${ }^{48}$ ». Si la première antithèse de l'organisme collectif des travailleurs était la dépendance radicale à l'égard du capital, il est également clair que le fonctionnement même de cet organisme implique une deuxième aliénation. En effet, il repose, à l'époque industrielle, sur le travail parcellaire de ses divers organes individuels, et est donc antithétique de l'individu intégral. Marx annonce le remplacement de l'individu morcelé par l'individu intégral, « qui sache tenir tête aux exigences les plus diversifiées du travail et ne donne, dans des fonctions alternées qu'un libre essor à la diversité de ses capacités naturelles ou acquises 49 ». À partir de ces deux aliénations (de l'organisme collectif des travailleurs par le capital, et de l'individu collectif par la division du travail), la dialectique marxienne de l'histoire cherche à rétablir la valeur d'une individualité collective, par l'intermédiaire de la communauté. La suppression de la première aliénation, c'est "l'avènement de la communauté50 ». Puis, les individus suppriment la division du travail et s'identifient à la communauté. Le travailleur collectif était une composition de parties, la communauté sera une individualité nouvelle et organique. Il est

46. Milne-Edwards, H., 1827, "Organisation ", Dictionnaire classique d'histoire naturelle, Bory de Saint Vincent et al., Beaudoin, 1822-1831; Milne-Edwards, H., Éléments de zoologie, Paris, Masson, 1843 ; Milne-Edwards, H., Leçons sur la physiologie et l'anatomie comparée des hommes et des animaux, tome I, Paris, Masson, 1857.

47. Marx, K., Le Capital, Quatrième section, Livre I, Ch. XIV, p. 259.

48. Hegel, G. W., 1821, Principes de la philosophie du droit, traduction R. Derathé, Paris, Vrin, 1975, $\mathbb{1} 187$.

49. Marx, K., Le Capital, Quatrième section, Livre I, Ch. XV, p. 350.

50. Calvez, J-Y., La pensée de Karl Marx, Paris, Esprit, Seuil, 1959, p. 513. 
intéressant de voir que l'association, au sens des socialistes français, disparaît, mais que le mot employé dans Les manuscrits de 1844 est le mot " communauté ». L'association, même si elle tend vers une structure sociale unifiée et dirigiste, implique des rapports entre individus. Ici, tout le but de Marx est de faire croire qu'on absorbe l'individu dans un nouvel individu représenté provisoirement par « la communauté », puis par l'humanité entière. Le sommet de la dialectique marxienne consiste justement dans le fait que « la communauté sera l'expression la plus complète de l'être personnel de l'homme, être social, parce que dès le premier rapport constitutif du réel, il est être générique ${ }^{51}$ ». Curieusement, Marx fait dépendre l'être générique de l'homme de l'activité vitale, par le moyen de la conscience : l'homme peut relativiser sa propre activité vitale $5^{2}$, et la rattacher à une existence proprement humaine.

L'homme fait de son activité vitale elle-même l'objet de sa volonté et de sa conscience. Il a une activité vitale consciente. Ce n'est pas une détermination avec laquelle il se confond directement. L'activité vitale consciente distingue directement l'homme de l'activité vitale de l'animal. C'est précisément par là, et par là seulement, qu'il est un être générique. Ou bien, il est seulement un être conscient, autrement dit sa vie propre est pour lui un objet, précisément parce qu'il est un être générique. C’est pour cela que son activité est une activité libre. ${ }^{53}$

Le travail aliéné renverse ce rapport de l'homme à une activité libre en rapport de dépendance. L'homme n'étant plus libre dans son activité de transformation de la nature, il n'est plus libre non plus dans la transformation de sa propre vie. " Donc, tandis que le travail aliéné arrache à l'homme l'objet de sa production, il lui arrache sa vie générique, et il transforme l'avantage que l'homme a sur l'animal en ce désavantage que son corps non-organique, la nature, lui est dérobé54. "L'aliénation fait que l'homme se heurte à toutes les limites de son individualité. Tout le but de la dialectique historique sera donc de rendre à l'homme sa vie générique par le moyen de la communauté qui relativise sa vie propre, et lui confère son

5I. Ibid., p. 513.

52. M. Henry (1991) souligne ce fondement de l'analyse de Marx dans l'activité vitale individuelle, la conscience, puis le passage à la vie générique étant des étapes ultérieures. Il écrit : "Tous les rapports qui se manifestent dans la société, qu'ils soient contradictoires ou non, renvoient en fin de compte à ce phénomène fondamental de l'activité productrice, c'est à dire encore à la vie. Toute l'œuvre ultérieure de Marx avec son aboutissement dans Le Capital se concentrera donc sur l'analyse et d'abord sur la reconnaissance des propriétés essentielles de la vie comme fondatrice de l'ensemble des rapports économiques" (Henry, M., Marx, une philosophie de la réalité, vol.1, Paris, Tel, Gallimard, 1991, vol.1, p. 217). M. Terestchenko (1992) commente ainsi cette affirmation de M. Henry : "C'est à un travail généalogique qui remonte des concepts à la vie, concepts qui n'ont aucune existence propre, aucun être en soi en dehors de leur réalité phénoménologique, que Marx nous convie. La philosophie de Marx est donc, pour Michel Henry, une incessante réduction phénoménologique des catégories de l'idéalité et de la pensée à la vie. C'est la vie des individus singuliers et uniques, la réalité humaine pratique concrète qu'il s'agit de révéler par delà son occultation et son objectivation conceptuelle et théorique dans l'idéologie. " (Terestchenko, M., Marx et l'ontologie de la vie selon Michel Henry, Les études philosophiques n 1-1992, Paris, PUF3, 1992, 101-116, p. 102)

53. Marx, K., Les manuscrits de 1844, I, p. 63.

54. Ibid., p. 63. 
humanité, dans un monde renouvelé, qui tend à être le milieu de vie de l'homme universel. La communauté devient alors le médiateur obligé entre l'homme et la nature, entre l'homme et le fruit de son travail, entre les individus eux-mêmes, afin de conférer à chacun non seulement le fruit de son travail, mais surtout cette humanité générique, universelle, dont parle Marx. Donc, la «vie productive" ne consiste pas d'abord à produire des marchandises, mais à "l'autoproduction de la vie générique humaine 55 ». Cette identité intentionnelle de l'homme individuel et de la communauté explique la position de Louis Dumont ${ }^{56}$, à propos de Marx.

La position de Marx, par rapport aux socialistes français est intéressante. Tandis que d'un côté il leur doit beaucoup, et va jusqu'à réclamer l'abolition de la propriété privée, de l'autre, il ne partage pas le moins du monde leurs réserves en ce qui concerne l'individu et leurs efforts vers une idée plus profonde de l'homme [...]. Le socialiste Marx croit à l'individu d'une manière qui n'a pas de précédent chez Hobbes, Rousseau, et Hegel, et même dirait-on, chez Locke ${ }^{57}$.

Mais peut-on vraiment parler de "réserves » des socialiste français, en ce qui concerne l'individu ? Nous avons vu que Saint-Simon, comme Fourier, tout en insistant sur le caractère associatif et unifié (voire mécanique) de la vie socio-économique, de l'activité sociale, conservent à terme les idées de rétribution inégale et de bonheur individuel. Le bien de l'individu ne se réduit pas chez eux à l'organisation sociale qu'ils préconisent. En réalité, Marx voit l'individu comme appelé à s'identifier à la société et au genre humain tout entier, au terme du processus dialectique. C'est probablement une des différences les plus fortes avec les socialistes français. Dès lors, il n'y a plus d'autonomie de telle ou telle dimension de la personne par rapport à la société. On ne peut plus non plus parler d'association, mais d'unité générique dont la communauté est médiatrice.

Quel est le sens de cette assimilation générale de l'individu à une communauté organique et vivante (Marx garde toujours, à l'arrière-plan, les analogies dans le domaine de la vie organique) ? Si on suit le texte du troisième manuscrit, on peut voir le caractère tout intentionnel de cette identification, et son achèvement dans l'unité. L'homme vivant est, au terme du texte cité cidessus, la totalité, mais " totalité idéale, existence subjective pour soi de la société pensée et sentie ». Finalement, c'est à l'unité de l'être social individuel réel et de la totalité, unité de la forme vivante réelle et de la conscience universelle, que vise la dialectique marxienne. Elle est, beaucoup plus

55. Rodrigo, P., Marx et la phénoménologie, Les études philosophiques n ${ }^{\circ} 1-1992$, Paris, PUF., 85-100, p. 90.

56. Dumont, L., Essais sur l'individualisme, Une perspective anthropologique sur l'idéologie moderne, Paris, Esprit, Seuil, 1991, p. 130-131.

57. Les Manuscrits de 1844 sont explicites sur cette « foi » de Marx en l'individu comme être social : «Ma conscience universelle n'est que la forme théorique de ce dont la communauté réelle, l'organisation sociale est la forme vivante. [...] L'individu est l'être social [...]. La vie individuelle et la vie générique de l'homme ne sont pas différentes" (1844, I, 90). 
puissamment que toutes celles qui l'ont précédée, dialectique d'unification. À ce niveau, la conception marxienne de l'État est explicitement celle d'un organisme vivant. Vieira de Mello écrit :

L'État, selon Marx est un organisme qui se donne pour but de concilier des intérêts contradictoires des diverses classes qui la constituent, autrement dit qui se propose de légaliser les rapports de domination ; il se place au-dessus de la société, et devient progressivement étranger à celle-ci. Par l'abolition de la société de classes cesse l'utilité d'un appareil destiné à préserver sa survie ${ }^{58}$.

Lénine, commentant un passage de Engels, écrit à ce propos :

La société qui réorganisera la production sur la base d'une association libre et égalitaire des producteurs, reléguera toute la machine de l'État là où sera dorénavant sa place : au musée des antiquités, à côté du rouet et de la hache de bronze $\mathrm{e}^{59}$.

Même si l'État marxiste est un organisme totalitaire et unificateur, il resterait donc chez ses promoteurs une nostalgie de la constitution d'une association libre.

Chez Marx, la coopération existe au début du processus dialectique, elle constitue une expérience nécessaire qui fait prendre conscience aux ouvriers de leur force de travailleur collectif ; mais au sens strict de collaboration professionnelle en vue de la production, elle est appelée à s'effacer progressivement dans la mesure où le travailleur doit devenir polyvalent. On ne peut pas établir le même genre de lien entre coopération et association que chez les auteurs précédents, puisque le mouvement historique de la dialectique impose une distance de temps entre les deux. Bien plus que chez Fourier, le processus dialectique inscrit dans le développement historique, consiste à produire une communauté nouvelle, unifiée parce qu'individualisée. Si on interprète les textes, on voit que la communauté nouvelle qui médiatise les rapports sociaux n'est autre que l'association des travailleurs. Si chacun s'identifie à la communauté et en reçoit son être social, cela suppose de se reconnaître associé et semblable aux autres, au point de former avec eux un seul individu (c'est la négation pure et simple de la personne humaine). Si la coopération entre individus doit subsister pour un temps, elle ne peut être que fortement intégrée dans cette individualité nouvelle, comme les relations d'interdépendance des organes dans l'organisme. On retrouve donc, comme chez Saint-Simon, une forme d'association créant l'organisme productif (et la priorité de cet organisme dans toute activité sociale), et comme chez Fourier, la production d'une société nouvelle, unifiée, intégrant dans son fonctionnement tous les aspects de la vie individuelle. Mais, la puissance de la dialectique hégélienne fait découvrir à Marx ce que ni Saint-Simon ni Fourier ne pouvaient découvrir : si la négation de l'aliénation rend l'organisme collectif à lui-même, la négation en lui-même de la division du travail l'identifie à chaque individu censé devenir indéfiniment adaptable et apte aux fonctions les plus diverses.

58. Vieira de Mello, S., Civitas maxima : origines, fondements et portée philosophique et pratique du Concept de supranationalité, p. 302.

59. Lénine, 1917, L’État et la Révolution, Paris, Editions sociales, 1967, p. 118. 
D'une certaine manière, comme dans les systèmes précédents, on retrouve à son terme l'individu. Mais, cette fois, ce n'est pas un individu qui a retiré de la société des avantages, tout en restant autre dans son être : c'est l'individu qui ne fait qu'un avec la société. L'unité, chez Marx, est dialectique, et elle est à ce niveau de synthèse. L'association est alors assimilée à l'organisme social, elle est censée être un fruit d'une évolution historique qui s'impose à tout individu. Cet organisme tentaculaire qui prétendait s'étendre à l'humanité entière n'a plus rien à voir avec une association proche des individus qui la composent. Il ne peut que se confondre avec l'émanation d'un pouvoir fort, mis en place à la suite de l'émancipation violente du groupe qui se considère comme aliéné.

\section{5. Éléments de conclusion}

Parcourant les écrits de Saint-Simon, Fourier et Marx, notre travail a donc cherché chez ces auteurs une intelligibilité du concept d'association. Chez les deux premiers, nous avons identifié le sens de mise en commun en vue de la production, avec une reconnaissance limitée des inégalités et de la propriété. Cette mise en commun tend à s'étendre chez Fourier à de nombreux aspects de la vie (habitat, loisirs, éducation, culture, communication...). Chez ces deux auteurs, l'association présuppose le moyen de l'organisation et devient le cadre normal d'activités de coopération. Il est intéressant de souligner qu'il existe une similitude entre les biologistes prédarwiniens du XIX ${ }^{\mathrm{e}}$ siècle et la naissance de la pensée socialiste. Avec Dugès (1831), on passe de l'association d'organismes élémentaires à la constitution fusionnelle d'une nouvelle individualité ${ }^{60}$. Le moyen terme permettant d'aboutir à un " tout " au plan biologique ou sociétal est la division du travail. Milne-Edwards (1857) ira plus loin en regardant l'individu comme résultat des divers organes et fonctions. Le « faire » n'est plus une propriété du vivant, mais il élabore le vivant, il crée une nouvelle structure, produit d'une réalisation commune. Donc, cette activité réalisatrice instaure une nouvelle unité organique, subordonnée à l'économie animale. Les deux étapes de l'histoire de la biologie que nous venons de citer nous semblent parallèles à l'élaboration de la pensée socialiste. La constitution fusionnelle d'une nouvelle individualité, d'un organisme sociétal «par le partage des activités et des occupations " (Dugès, 1831) semblerait faire écho aux projections de Fourier dans la Théorie des quatre mouvements (1808), ou Le mouvement industriel et sociétaire $(1829)^{61}$. L'idée de communautés industrielles, unifiées et autosuffisantes, reposant sur une saine organisation du travail et le bon usage du dynamisme des passions, caractérise le socialisme fouriériste. Il semblerait alors que, dans les trente premières années du dix neuvième siècle, l'histoire de la pensée oscille entre l'exaltation de l'individu,

60. Dugès, A., Mémoire sur la conformité organique, Montpellier, Ricard, 1831.

6r. Fourier, C., Le mouvement industriel et sociétaire, Paris, Société pour la propagation et la réalisation de la théorie de Fourier, 1829. 
capable de créer l'évolution, et la tendance vers de nouvelles individualités, organiques et sociétales.

Le primat de l'activité réalisatrice, comme produisant l'organisme vivant dans son unité et le subordonnant à l'économie animale, se retrouverait beaucoup plus dans l'œuvre de Marx. Pour Marx, c'est le travail et l'échange qui produisent l'homme, ou plutôt l'objectivent en une nouvelle nature, et élaborent la société. Au terme de la démarche, la société sera une nouvelle individualité, l'expression la plus complète de l'être personnel de l'homme, de l'être social (Manuscrits de 1844). Ainsi, le concept d'association semblerait pratiquement disparaître dans cette recherche d'identification mutuelle entre l'individu et la société. Toutefois, si on interprète correctement les textes, la communauté paraît associée ou plutôt fédérée par les événements historiques qui la font s'ériger en médiateur entre l'homme et son milieu de vie. Le point sur lequel la pensée de Marx diffère complètement de celle des socialistes français (et même des naturalistes comme Milne-Edwards) est la division du travail en lien avec l'organisation sociale. Essentielle selon eux pour penser l'association, elle doit être supprimée dans le " devoir être " de la société communiste marxiste. La signification du concept d'association passerait donc du sens d'engagement de l'individu dans la société, supposant mise en commun limitée, organisation collective des productions et création d'unité politique, à l'idée d'organisme social constituant les citoyens dans leur individualité sociopolitique. Marx représente donc un moment de la pensée politique où la communauté humaine s'érigeant en organisme générique l'emporterait sur l'association, au sens ou nous l'avons définie. À partir du moment où l'expérience de coopération libre et volontaire entre des personnes humaines est trop relativisée à l'existence de structures organisées et collectivistes (Fourier) ou à la formation idéale d'un nouveau corps social organique (Marx), on risque fort les dérives du socialisme étatique et totalitaire. Une véritable association serait donc pleinement respectueuse de la libre coopération et de l'activité commune des membres qui s'y engagent, elle en dériverait plus qu'elle ne l'encadrerait, le concept lui-même impliquant toute une série de degrés, des corps intermédiaires structurant la société.

Il est intéressant de noter que tous les totalitarismes sont sortis de visions politiques trop unifiantes, c'est à dire, insistant sur l'unité entre la partie (l'individu) et le bien du tout-un. Si la pensée marxienne a connu les déviations du totalitarisme, c'est parce qu'identifiant l'homme et la communauté sociale dans l'unité, elle ramenait pratiquement de façon totalitaire tout acte individuel à un acte communautaire. Karl Popper, dans The Open Society ${ }^{62}$, considère $\mathrm{La}$ République de Platon comme la première explicitation du totalitarisme, parce que « ce que Popper tient pour totalitaire dans la pensée de Platon, c'est l'idée que serait juste ce qui est dans l'intérêt du tout, de la cité (bonne), et que la justice, au fond, c'est l'inégalité, la hiérarchie (chacun à sa place) ${ }^{63}$. » Dans la

62. Popper, K., The Open Society, Conjectures et réfutations : la croissance du savoir scientifique, traduction M.-I. et M.-B. de Launay, Paris, Bibliothèque scientifique Payot, 1985.

63. Boyer, A., Libéralisme, démocratie et rationalité, in « L'âge de la science, Éthique et philosophie politique ", Paris, Éd. Odile Jacob, 1988, 105-131, p. 121. 
perspective d'une autosuffisance morale de l'individu, Popper verrait l'association comme organisation impliquant l'interdépendance sociale. En ce sens, Popper oppose aux théories unifiantes de la souveraineté, un « libéralisme réformiste et activiste ${ }^{64}$. $"$ Il représente une tendance de la critique contemporaine des concepts collectifs traduisant l'unité de l'État ou d'une classe : c'est un individualisme méthodologique, qui refuse toute forme de holisme. Un intérêt, pour nous, de cette critique de Popper est que cet épistémologue voit l'interdépendance des hommes et des nations comme compatible avec la liberté d'action des individus et leurs fins propres. Par ailleurs, il reconnaît que l'action humaine ne se limite pas au seul cadre du conditionnement physique et biologique. On retrouve toujours l'idée selon laquelle une association humaine échappe au déterminisme biologique, à l'organicisme, parce qu'elle intègre un ordre à des fins « individuelles" ou "personnelles", selon les auteurs.

Une autre critique contemporaine des structures politiques unifiées est celle de Claude Lefort : elle est de nature à la fois historique et phénoménologique. Lefort écrit : "L'essor du totalitarisme nous met en demeure de réinterroger la démocratie ${ }^{65} »$. Comme Tocqueville opposait la société aristocratique à la société démocratique ${ }^{66}$, Lefort oppose aussi la société totalitaire à la société démocratique. À la mutation politique qu'est le totalitarisme, que Lefort considère comme reflétant un "idéal radicalement organiciste ${ }^{67}$ ", il oppose la société démocratique qui suppose la liberté d'opinion, d'expression, d'association. La démocratie est la société historique par excellence puisqu'elle dépasse la détermination organiciste de l'état totalitaire unifié68. Du côté de la forme, elle "accueille et préserve l'indétermination. »

64. Ibid., p. 128.

65. Lefort, C., Essais sur le politique, Paris, Esprit, Seuil, 1986, p. 21.

66. Tocqueville, A., 1856, L'Ancien Régime et la Révolution, Paris, nrf, Gallimard, 1967, p. 265.

67. Lefort, C., Essais sur le politique, p. 23.

68. Chêne, J., Penser le politique, in "L'âge de la science, Éthique et philosophie politique », 133-150, Paris, Éd. Odile Jacob, 1988. 